\title{
Online Learning and Social Norms: Evidence from a Cross-Cultural Field Experiment in a Course for a Cause
}

\author{
${ }^{\alpha}$ Ji Yong Cho, ${ }^{\beta}$ Yue Li, ${ }^{\beta}$ Marianne E. Krasny, ${ }^{\beta}$ Alex Russ, ${ }^{\alpha}$ Rene F. Kizilcec \\ ${ }^{\alpha}$ Cornell University, Department of Information Science \\ ${ }^{\beta}$ Cornell University, Department of Nature Resources and the Environment
}

Corresponding author: jc3374@ cornell.edu

\begin{abstract}
Online courses are increasingly used to scale up professional learning. However, engaging people throughout a course and encouraging them to take actions afterward is challenging, especially with culturally diverse participants whose response to encouragement may vary. Social norms can serve as a strong encouragement to participants, but they are mostly unobservable in online courses. We therefore tested how communicating three established kinds of social norms impacts behavioral outcomes and how the effects vary across different cultures. Findings from a randomized controlled field experiment in a Massive Open Online Course entitled Nature Education show that both the type of norm message (descriptive; dynamic; injunctive) and the cultural context (China; US) influence how the intervention improves course outcomes: US participants' completion rate rose $40 \%$ following an injunctive norm message stating that participants ought to conduct nature education, while other norm messages were ineffective for both US and Chinese participants. Regardless of the social norm message, nearly all participants engaged in nature education activities within three months after the course ended. Communicating social norms can impact participants' behavior in the course and beyond, which can offer a strategy for supporting online professional learning and tailoring messages to different cultural contexts.
\end{abstract}

Cho, J. Y., Li, Y., Krasny, M., Russ, A., Kizilcec, R., (2021) Online Learning and Social Norms: Evidence from a Cross-Cultural Field Experiment in a Course for a Cause. Computer-Based Learning in Context, 3 (1), 18-36.

Keywords: Online intervention, Social norms, Professional development, Field experiment, Cultural differences.

\section{Introduction}

Today's digital knowledge economy obliges workers to continuously update and expand their skill sets. This is contributing to the adoption of online professional learning as a means for access to state-of-the-art knowledge without the time and location constraints of in-person seminars (Karnouskos, 2017; Egkiffstein \& Ifenthaler, 2017; Joyner et al., 2020). Moreover, efforts to curb the spread of the COVID-19 virus during the 2020 global pandemic required most educational offerings to be available online, which resulted in an unexpected boost in demand for online learning (Crawford et al., 2020; Kizilcec et al., 2021). Despite the growing interest and use of online content for professional development, we have a limited understanding of how to support professionals in staying engaged with an online course. Research on Massive Open Online Courses (MOOCs), which emerged as a popular source for professional development content, has found high rates of attrition including among employed learners and those motivated by professional aspirations (Kizilcec \& Schneider, 2015; Kizilcec \& Halawa, 2015). It is therefore important to examine ways to help professionals stay engaged until the end of a course, so that they can reap the benefits in terms of career advancement.

Professionals taking online courses for career development are expected to transfer learned skills and knowledge into work practice (Napier, Huttner-Loan, \& Reich, 2020). For example, the Course for a Cause model (Krasny et al., 2019) emphasizes building a learning community to foster each other's professional growth and create real-world applications based on what was covered in the course. Through online courses, professionals with similar interests and career goals can connect worldwide to build a community that seeks to augment learning by sharing experience and knowledge from their workplace. For practice-based professional development, where learning is situated in practice (Ball \& Cohen, 1999), such as teacher education, environmental education, or medical practitioner training, individuals' actions outside of the course matter the most. To support this type of professional growth, we need to go 
beyond research that examines challenges with course completion rates, and study intervention strategies that can help build professional communities and encourage individuals to change their professional practices.

To stimulate engagement in learning during and after the course, we turn to the established strategy of highlighting social norms to shape human behavior (Cialdini, 2003). Social norms can be communicated in different formats of messages. The message can describe what behavior most people conduct (descriptive norm messages, e.g., 'most people eat healthy food.'), state what behavior people should conduct (injunctive norm messages, e.g., 'people should eat healthy food.'), and what behavior a growing number of people conduct (dynamic norm messages, e.g., 'more people eat healthy food nowadays.'). Although classic social norm messages have not yet been tested in online learning environments, prior research shows that communicating norms can have a positive impact on learning behavior. For example, reputation systems and badges in discussion forums, which set a norm that certain learning behavior is encouraged in the course, increase engagement in courses (Anderson, Huttenlocher, Kleinberg, \& Leskovec, 2014; Coetzee, Fox, Hearst, \& Hartmann, 2014), and a learning analytics dashboard that displays other course participants' progress increases course completion rates (Davis et al., 2017).

A critical limitation in our scientific understanding of social norms interventions is which types of messages are effective for participants in different cultural contexts, since the vast majority of social norms intervention research has been conducted in Western countries (Henrich, Heine, \& Norenzayan, 2010). Cultural psychologists have shown that culture affects how people perceive and behave in response to social norms (Fischer, 2006; Trongmateerut \& Sweeny, 2013). In fact, people consider one type of social norm more important than another depending on their cultural background (Culiberg \& Elgaaied-Gambier, 2016; Walker, Courneya, \& Deng, 2006). Given the international and culturally diverse audience in many online learning contexts today, most notably in Massive Open Online Courses (MOOCs) that attract people from around the globe, crafting social norm messages that can be universally effective presents a daunting challenge. For example, how might norm messages be tailored to specific cultures to shape behavior? The cultural diversity in the online learning environment offers a rare opportunity for advancing our understanding of how people in different contexts respond to norm messages and similar behavioral science interventions (Kizilcec \& Cohen, 2017; Kizilcec et al., 2020).

This study contributes to our knowledge about what type of social norm messages can encourage professionals across cultures to persist in their career development efforts. In particular, we examine the degree to which norm messages can motivate people in different cultures to persist in online learning, to engage in their peer community, and to subsequently translate learned skills into work-related actions. We conducted a randomized controlled field experiment in a Nature Education MOOC, an exemplary online course for practice-based professional development with the goal of enhancing participants' capacity as educators to conduct educational activities that connect their audience with nature in local communities. We then followed up with participants after the course ended to observe the extent to which they implemented educational practices in their local communities. We compare how the social norm interventions affect participants from an individualist culture (the United States) and a collectivist culture (China) to sharpen our understanding of which type of social norm intervention should be deployed in each context. Our findings reveal that both the type of norm message (descriptive norm; dynamic norm; injunctive norm) and the cultural context (China; US) determine whether the intervention changes participants' behavior. We discuss how this begins to advance our understanding of effective strategies for supporting online professional development and how they need to be adapted in response to different cultural backgrounds.

\section{Background}

2.1 Social Norms Interventions in Behavior Change. Social norms interventions are an effective approach for changing human behavior. Prior research on social norms intervention has focused on promoting prosocial behavior such as pro-environmental actions (Farrow, Grolleau, \& Ibanez, 2017). In these interventions, norm messages communicate what behavior most people engage in (a descriptive norm) or what behavior most people morally endorse in society (an injunctive norm) (Cialdini, 2009). Recently, norm messages have begun to draw attention to temporal changes in people's behaviors; for example, noting that the number of people engaging in a target behavior has increased over time (a dynamic norm) (Sparkman \& Walton, 2017).

If a target behavior is already prevalent in the community, we can motivate behavioral change by directing attention to the prevalence of that behavior. This can be achieved by highlighting a descriptive norm indicating what most others do. For example, Goldstein et al. (2008) found that more hotel guests reused towels if they received a descriptive norm message that stated that the majority of the guests reused their towels, instead of a simple request to reuse towels that emphasized the importance of saving the environment. Descriptive norms have also been used to correct a prevailing misconception that most college students engage in undesirable behaviors such as binge drinking 
and smoking, which decreased college students' drinking and smoking by clarifying that these behaviors are actually rare in college (see Berkowitz, 2004, for a review).

If a target behavior is not prevalent, an injunctive norm calling attention to what people should do rather than what people currently do can promote the target behavior. For example, Cialdini et al. (2006) compared injunctive norm messages and descriptive norm messages that were intended to reduce petrified wood theft in Arizona's Petrified Forest National Park. The injunctive norm message, "Please don't remove petrified wood in the park," was more effective in reducing wood theft than the descriptive norm message, "Many past visitors have removed the petrified wood from the park, changing the state of the Petrified Forest." Similarly, a lab study found that presenting an injunctive norm message, "Choose a sustainable cup" instead of a descriptive norm message, "Approximately $25 \%$ are choosing a sustainable cup," significantly increased participants' intention to use reusable mugs in a cafe (Loschelder, Siepelmeyer, Fischer, \& Rubel, 2019).

If a target behavior is uncommon or rare, dynamic norms that spotlight a trend in the adoption of the target behavior can be effective in persuading people to join the trend. Past research has found that presenting other people's behavior change over time promotes the target behavior even if a change takes place within a minority of people (Mortensen et al., 2019). In such cases, a dynamic norm message has been reported to be more effective than a descriptive norm message. For example, a dynamic norm message indicating that people have begun eating less meat in recent years increased the number ordering a meatless lunch in a cafeteria significantly more than a descriptive norm message that simply stated that a minority of the people are presently making an effort to reduce meat consumption (Sparkman \& Walton, 2017). However, there is not enough evidence to determine if a dynamic norm message may also be more effective than an injunctive norm message when a target behavior is uncommon.

Overall, the literature on social norms interventions recommends that norm messages be tailored based on the prevalence of the target behavior in the immediate community. However, in the context of a large online course where community norms remain mostly unobservable to participants, it is unclear what type of social norm message would be effective. In particular, information about the target behavior, such as practicing learned skills outside of the course and conducting education practices is not readily available to course participants. The present study therefore compares descriptive, injunctive, and dynamic norm messages to provide novel insights into the effective use of norm interventions in support of career development using online courses. Thus, our study addresses the following research question:

RQ 1. How do different types of norm messages affect participants' initial post-intervention beliefs, completion rate, and engagement in the course?

2.2 Social Norms Interventions in Different Cultural Contexts. Social norms interventions leverage individuals' tendency to conform to group behavior and expectations. Research in cultural psychology has argued that the impact of social norms on behavior would vary across cultures (Fischer, 2006). In individualistic cultures like the US, people view themselves as independent actors who value uniqueness, while in collectivist cultures like China, people view themselves as inseparable parts of a social whole, and strive to achieve social acceptance and harmony (Hofstede, 1980; Oh, 2013; Triandis, 1995). Research has suggested that people from collectivist cultures tend to be more influenced by social norms (Trongmateerut \& Sweeny, 2013). Another line of work has proposed that the strength of social norms is dependent on how tight or loose the culture is. Countries with tight cultures including China "have strong norms and a low tolerance for deviant behavior," while less controlled or "loose" societies including the US "have weak norms and a high tolerance for deviant behavior" (Gelfand, 2012; Gelfand, Harrington, \& Jackson, 2017). In keeping with the tightness-looseness theory of culture, Chinese participants are more sensitive to norm-violating behavior than US participants according to neurobiological evidence (Mu, Kitayama, Han, \& Gelfand, 2015). Overall, this work suggests that it is likely that social norms are more influential in shaping behavior in collectivist and tight cultures than in individualistic and loose cultures.

Prior research has shown that the effects of social norms manifest in different ways in collectivist and individualistic cultures. For instance, Thai participants (collectivists) were more swayed by subjective norms to engage in whistle-blowing than US participants (individualists) when they encountered misconduct in the workplace (Trongmateerut \& Sweeney, 2013). Culiberg and Elgaaied-Gambier (2016) also found that the perception of how others expect someone to act (injunctive norm) mediates the relationship between pro-environmental norms at the country level and individual intentions to take pro-environmental actions in Slovenia (a collectivist culture) but not in France (an individualistic culture). Another study that compared two culturally distinct ethnic groups in Canada--British Canadians who are individualists and Chinese Canadians who are collectivists---found that perceived acceptance of playing the lottery (injunctive norm) is predictive of male British Canadians' intentions to play the lottery, but perceived prevalence of the behavior (descriptive norm) is predictive of male Chinese Canadians' lottery playing intentions (Walker, Courneya, \& Deng, 2006). The study further demonstrated an indirect effect of norms on 
real-world behavior by showing that participants who intended to play the lottery were also more likely to spend money on buying lottery tickets.

How norm messages influence individual behavior in different cultures has received scant researcher attention. A few studies that focused on the interaction between a descriptive norm message (prevalence or not) and an injunctive norm message (approval or disapproval) suggest that cultural differences are minimized when both types of messages are combined. Bresnahan and Zhuang (2016) found that the message describing low prevalence of smoking (descriptive) and disapproval of smoking (injunctive) increased factory workers' resistance to social smoking in China (a collectivist and tight culture). Likewise, Smith et al. (2012) found that both Chinese and UK undergraduate students reported the highest rates of intention to engage in energy conservation when they were told the majority of their peers were conducting and endorsing such behavior. However, these studies do not show if the type of norm message used (descriptive, injunctive, dynamic) has different effects on different cultural groups, and if so, the nature of those effects.

Overall, the literature on cultural psychology suggests that collectivists are more attuned and susceptible to social norms than individualists. Several studies have shown empirically that collectivists and individualists behave differently based on their perceptions of the social norms in an environment. However, the extent to which these findings translate to social norms intervention, where a message is sent to communicate a social norm in order to change a target behavior, remains an open question. The intervention effects may or may not differ between collectivist and individualist cultures based on the type of norms invoked in the message. The current study addresses this question by comparing descriptive, injunctive, and dynamic norm messages in their effect on Chinese and US participants' behavior in the context of online learning. We therefore address the following research question:

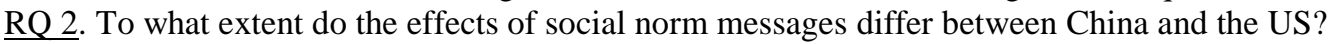

2.3 Social Norms Interventions in Online Learning. In contrast to the effects on most target behaviors in prior studies, the effects of norm interventions on complex behaviors that require sustained action beyond a single behavior produce more mixed results. For example, a descriptive norm message stating the number of people who offset their carbon emissions failed to influence people's behavior or interest in spending money to offset their own air travel emissions (Tyers, 2018). Yet another study found that a descriptive norm message describing the percentage of households on the same street participating in food waste recycling compared with the average for their neighborhood had a significant positive impact on the recycling participation that cost time and maybe an inconvenience (Nomura, John, \& Cotterill, 2011). Professional development requires sustained effort over time in sharpening skills and obtaining up-to-date knowledge. In teacher education in particular, conducting education activities involves multiple actions including defining target audiences and learning objectives, developing lesson plans, and delivering learning activities, all of which must be considered in measuring changes in educational practice (Yue Li \& Krasny, 2019). Adopting new instructional methods or changing existing practices requires educators to build confidence, skills, and knowledge as well as a partnership with fellow educators, students, community members, and local facilities (Green \& Somerville 2015). There is little evidence for the kind of norm message, if any, that can encourage people to carry out these kinds of complex behaviors.

Prior research has tested the influence of social norms in the context of MOOCs using indirect intervention approaches. One study used a learning analytics dashboard to convey a descriptive norm of how previously successful learners were behaving in the course (Davis et al., 2017). This approach facilitated an upward social comparison, which led to an increase in completion rate by $22 \%$ and social course engagement, which refers to student activities within the course. Two other studies have experimented with communicating injunctive norms via badges in discussion forums (Anderson et al., 2014; Coetzee et al., 2014). Badges are visible to other course participants as a sign of exemplary behavior, such as answering peer questions in a timely manner. The studies found that introducing badges increases course participants' participation in forum discussions. Another study tested the effects of email messages about how MOOC discussion forums are generally used on social course engagement (Kizilcec, Schneider, Cohen, \& McFarland, 2014). Messages describing the forum as a communal place for social exchange (collectivist) or as a convenient feature to get one's own questions answered (individualist) backfired and reduced social course engagement compared to a neutral message reminding course participants of the existence of a forum. Taken together, prior studies on social norms in MOOCs employed a variety of intervention types and found positive as well as negative results.

It is unclear whether a normative message is an effective approach to encourage a target behavior such as learning in a MOOC. Norm interventions target individuals' social desire to fit in a community, but MOOC participants are typically only weakly tied to each other (Clinnin, 2014; Kellogg, Booth, \& Oliver, 2014; Kizilcec et al., 2014). Compared to in-person classrooms that inherently bring students together as a learning community, MOOCs are selfdirected classes where individual course participants proceed with course materials "without the immediate presence 
of a roomful of classmates" (Eisenberg \& Fischer, 2014). In this sense, a norm message based on the course's learning community, to the extent that it exists, may not motivate course participants to act differently. Instead, a norm message could appeal to the course participants' local community, but this raises the question of how to craft a suitable norm message for course participants who are globally distributed. To choose the most effective type of norm message for an intervention, researchers or practitioners must identify the current status of the target behavior, including how prevalent the target behavior is at present and how its adoption has changed over time. This challenge is especially pronounced when a target behavior needs to be carried out in physical settings, such as conducting educational activities in local communities, as MOOCs can hardly provide situational information about the target behavior due to the "screen-based nature of the presentation channel" (Clinnin, 2014). Therefore, instead of starting by diagnosing the prevalence of a target behavior, all three types of norm messages, descriptive, injunctive, and dynamic, can be tested simultaneously. In this way, it can be inferred which norm message motivates course participants to conduct the target behavior outside of the course or put the learned knowledge into practice despite the absence of information about the community which course participants relate to, and the prevalence of the target behavior in that community. Evaluating the impact of an intervention on participant engagement in the course and beyond presents a set of challenges. First, engagement can be quantified in many ways within the course using log data collected from online learning platforms (DeBoer, Ho, Stump, \& Breslow, 2014). Course completion is one of the most commonly used outcome measures in (online) education research, and it can serve as a summary indicator of high academic engagement. Still, depending on the course pedagogy, it can reflect different types and levels of engagement (Kahan, Soffer, \& Nachmias, 2017). For example, courses grounded in social learning employ in-platform discussion boards (Skrypnyk, Joksimović, Kovanović, Gašević, \& Dawson, 2015; Zhang, Skryabin, \& Song, 2016), social media (DuBois, Krasny, \& Russ, 2019), and study groups (Brinton et al. 2014; Krasny et al., 2018). Course participants' engagement in these channels often counts as one of the requirements for course completion. Other courses grounded in constructivist pedagogy may instead use frequent project submissions to provide course participants with opportunities to construct knowledge individually (Barak \& Watted, 2017) or with peers (Spoelstra, Van Rosmalen, \& Sloep, 2014). Second, measuring longer-term learning behaviors is even more challenging. This is especially true for behavioral outcomes after course completion. Only a few studies have measured MOOC participants' behavioral outcomes in the long-term from the perspective of knowledge transfer (Chen, Davis, Hauff, \& Houben, 2016), knowledge co-creation (Krasny et al., 2018), teaching practice (Napier, Huttner-Loan, \& Reich, 2020) and career advancement (Wang, Paquette, \& Baker, 2014). We contribute to this nascent area of scholarship by addressing the following research question:

$\underline{\text { RQ 3 }}$. How much does a practice-based online course encourage post-course engagement among its participants?

Our goal in this study is to advance a scientific understanding of the effectiveness of different types of norm messages embedded in a practice-based MOOC for professional development in increasing participants' engagement in the course and the implementation of activities in their communities. Moreover, this study takes a cross-cultural approach to examine how the impact of the interventions varies across Chinese and US contexts.

\section{Methods}

3.1 Context and Participants. This study was conducted in a Nature Education MOOC. It was a practice-based course designed for pre- and in-service teachers and non-formal educators interested in nature education worldwide. Course participants were expected to exchange ideas about educational activities with each other in structured discussions and to develop an actionable lesson plan for their local setting. This course allowed us to examine the impact of social norm interventions not only on in-course engagement data including discussion and course completion, but also on post-course nature education activities reported in a follow-up survey.

The course was offered by Cornell University's civic ecology lab on the edX Edge platform from April 9th to May 14th, 2019. This five-week course was offered in both English and Chinese. The course materials comprised video lectures and readings for self-paced learning, and weekly webinars with guest speakers and social media groups for informal peer interaction. The video lectures were narrated in English and provided closed captioning in Mandarin Chinese. The course also had a group of Chinese TAs to summarize English readings into Chinese and facilitate study groups to assist Chinese participants with understanding course materials. Participants registered for the course via a survey and were then enrolled to the edX Edge course platform. To ensure that the course is accessible to as many people as possible, participants could choose to either pay the standard registration fee $(\$ 60)$, any amount they could afford, or enroll for free. Registrants were randomly assigned into four groups based on their registration information to achieve covariate balance. Among 648 registrants, a total of 590 participants enrolled in edX Edge (150 control; 142 descriptive norms; 146 dynamic norms; 152 injunctive norm). Participants were 37 years old on average (SD = $11, M d n=36), 76 \%$ women, $84 \%$ had a bachelor's or higher degree, and $78 \%$ had work experience in educational 
practices in the field of nature education. Most participants were nationals of China (52\%), the US (29\%), Nigeria (3\%), and Canada (2\%), out of 48 countries overall. The most frequently reported occupations were non-formal educators $(30 \%)$, non-academic professionals including corporate and independent employees $(24 \%)$, university students $(11 \%)$, and $\mathrm{K}-12$ teachers $(10 \%)$.

Our comparative analyses of participants in China and the US focus on a subset of 478 individuals ( 306 in China). Participants in both China and the US are predominantly female ( $79 \%$ female in China, $81 \%$ female in the US; $21=$ $0.16, p=0.69$ ), but US participants were 6 years older on average (41 yrs on average $[S D=13, M d n=39]$ in the US vs. 35 in China $[S D=9, M d n=36] ; t 1=-5.81, p<.001$ ), more educated (90\% in the US vs. $81 \%$ in China with a bachelor's or higher degree; $2(1)=5.31, p=.02$ ), and had more work experience in nature education practice ( $87 \%$ in the US vs. $70 \%$ in China; $21=15.35, p<.001$ ). Chinese participants self-reported their occupations as nonacademic professionals (30\%), non-formal educators (26\%), university students (11\%), K-12 teachers (8\%), university professors or researchers (7\%), and parents (5\%), while US participants reported theirs as non-formal educators (46\%), K-12 teachers (15\%), other professions (14\%), and university students (5\%).

3.2 Procedure. We used course registration survey data for criteria-based randomization to ensure balance in the covariate distribution (Baiocchi \& Kizilcec, 2018). Participants were shown one type of social norm message in video lectures according to their assigned condition during the course. No normative message was shown to participants in the control condition. Three months after the course ended, a follow-up survey was sent to all course participants asking whether they have engaged in nature education practices since taking the course.

3.3 Social norms intervention. We crafted three types of normative messages modeled after examples in prior work that used social norm messages as interventions (e.g., Goldstein, Cialdini \& Griskevicius, 2008; Cialdini et al., 2006; Sparkman \& Walton, 2017). However, unlike prior studies that used a specific percentage number (e.g., "almost 75\% of guests") in the normative message, our study used more abstract terms such as "millions of people," "more and more people," and "many people." This was because we could not provide an accurate number of people conducting nature education activities worldwide for a global audience. We embedded the following messages in the video as shown in Figure 1: "Millions of people are conducting nature education activities in their communities, organizations and families. You can do it too!" (descriptive norm message), "More and more people are starting to conduct nature education activities in their communities, organizations and families. You can join this movement!" (dynamic norm message), and "Many people believe that everyone should conduct nature education activities in their communities, organizations and families. You can do it too!" (injunctive norm message).

The normative message was embedded in a short video lecture. The video began by showing a graphic and reading the normative message, continued with course content, and ended with showing the same message with the graphic again in full screen (Figure 1). Similar to other video lectures, Chinese translation was provided as closed captions. The video containing the treatment message was immediately followed by a value-relevance activity to reinforce the normative message by having participants connect the act of conducting nature education to their self-concept (Kizilcec, Saltarelli, Reich, \& Cohen, 2017). The value-relevance activity first asked participants to report "What is most important to you? (choose from a list of options such as relationships with friends or family, and health and wellbeing)" and then "How does conducting nature education reflect and contribute to what is most important to you?" The normative message was presented one more time in week 5 without the value-relevance activity. 


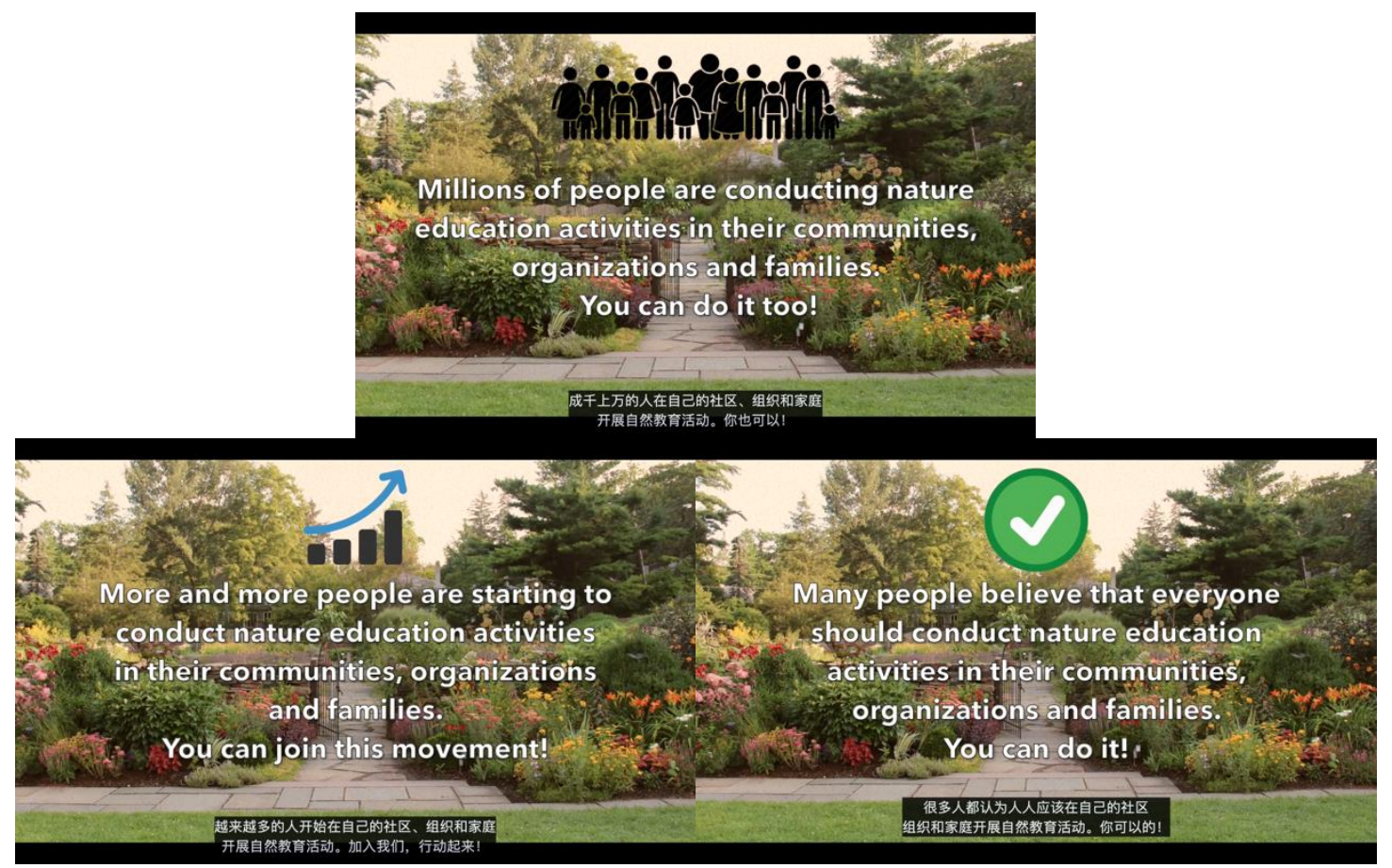

Figure 1. Norm Messages Embedded in Video Lectures: descriptive (top), dynamic (bottom left), and injunctive (bottom right) norm message. Chinese translation was also provided in the videos for Chinese speakers. No message was included in the video for participants in the control condition.

3.4 Measures. To assess the immediate effects of the intervention on participants' beliefs, a four-question survey was placed immediately following the first manipulation in week 2 of the course materials (i.e., the video lecture and valuerelevance activity) on the course platform. The survey asked two manipulation check questions ("What percentage of people around the world are conducting nature education?"; "What percentage of people think that conducting nature education is important?") and two motivation questions ("How likely are you to conduct nature education outside of the course?"; "How likely are you to complete this course from start to end?") on a scale from 0 to $100 \%$.

To measure the impact of the intervention, we focus on course completion and social course engagement. First, participants' course completion was measured by whether they received a certificate of completion. This required submitting a final project, creating at least one post and one comment to peer's posts on each of the four weekly discussion boards, and making at least one reflection on webinars including nine English ones and two additional Chinese ones during the course. The overall course completion rate was 60\%, 63\% for Chinese participants and 59\% for US participants. Second, social course engagement was measured by the individual's total number of posts, webinar reflections, and comments in the course. It did not include course participants' engagement or activity outside of the course. The weekly discussion questions asked participants to reflect on their own experience and how to integrate learned ideas into their practices. Participants were also asked to comment on at least one other fellow participant's post each week. The webinar reflections asked participants to summarize the main ideas from any webinars and connect ideas from webinars to the course lectures and readings as well as their own practices. Although we had minimum requirements for certificates, many participants went beyond this minimum to engage in the discussions with fellow participants. The total number of the posts, comments and webinar reflections therefore provides an indicator of participants' social course engagement. The average social course engagement across all participants was 8.78 posts $(S D=7.37, M d n=10) ; 9.11(S D=7.46, M d n=10)$ for Chinese participants and 8.19 $(S D=6.49, M d n=9)$ for US participants. Social course engagement was correlated with course completion as course participants were required to participate in in-course discussion to receive a certificate (Pearson $r(476)=$ $.77, p<.0001)$.

The follow-up survey, which was sent to all participants three months after the course had ended, asked about 12 different kinds of nature education activities that participants may have conducted since taking the course (Table 1). For each activity, participants were asked to answer 'yes' or 'no' to report on their recent activity. The survey was 
completed by 131 out of all 590 course participants (22\% response rate). About $82 \%$ of respondents had completed the course. Among the 356 participants who completed the course, $30 \%$ responded to the survey. Respondents were from China (54), the US (43), Nigeria (6), and several other countries (28). They were relatively evenly distributed across experimental conditions: 31 control, 29 descriptive norms, 36 dynamic norms, and 35 injunctive norms. Although more than half of the course participants were experienced in nature education, there were also inexperienced participants who completed the survey. Approximately 23\% (vs. 19\%) of participants with (vs. without) work experience in nature education completed the survey.

Table 1. Long-Term Nature Education Outcomes: Distribution of Responses from a Follow-Up Survey About Which Nature Education Actions Participants Have Taken Three Months After Taking the Course. ${ }^{1}$

\begin{tabular}{lr}
\hline Actions taken three months after course completion & Checked n (\%) \\
\hline I integrated nature education into other activities. & $108(82.44 \%)$ \\
I involved my family and friends in nature education activities. & $97(74.05 \%)$ \\
I enhanced my current nature education activity or activities. & $84(64.12 \%)$ \\
I implemented the lesson plan I developed during the course. & $82(62.60 \%)$ \\
& \\
I initiated a new nature education activity. & $82(62.60 \%)$ \\
I used new teaching approaches in my current nature education & $77(58.78 \%)$ \\
activity or activities. & $70(53.44 \%)$ \\
I participated in a new nature education activity organized by & \\
others. & $65(49.62 \%)$ \\
I expanded locations of my current nature education activity or & \\
activities. & $52(39.69 \%)$ \\
I kept in touch with some course participants. & $47(35.88 \%)$ \\
I trained other educators in nature education. & $39(29.77 \%)$ \\
I collaborated with other course participants to conduct a nature \\
education activity.
\end{tabular}

3.5 Data Analyses. We use OLS regression with covariates and report robust standard errors. Robust standard errors are used because the assumption of homoscedasticity of variances is usually violated in web data. No multiple testing corrections were applied to regression models given that experimental differences were evaluated in one model specification. Participants' self-reported background information, which was also used for balanced random assignment, was standardized (i.e., z-scored) and added to all regression models as covariates. The assigned norm condition was dummy coded (0/1) and not standardized. These covariates are age (numeric), gender (binary), educational level (binary; Bachelor's or higher degree), work experience in nature education (binary), teaching a nature education program or course at the time of course registration (binary), knowing anyone working in the nature education field (binary), and nationality (Chinese, US, or other). Nationality was omitted in country-specific analyses. We fitted the same multiple regression model with covariates to each outcome measure separately. The regression model was specified as follows:

$$
\begin{aligned}
Y_{i}=b_{0}+ & b_{1} X_{\text {descriptive }, i}+b_{2} X_{\text {dynamic }, i}+b_{3} X_{\text {injunctive }, i}+b_{4} X_{\text {isFemale }, i} \\
& +b_{5} X_{\text {age }, i}+b_{6} X_{\text {isBaOrHigher }, i}+b_{7} X_{\text {hasWorkExp }, i} \\
& +b_{8} X_{\text {knowsSomeone }, i}+b_{9} X_{\text {isInProgram }, i}+\varepsilon_{i}
\end{aligned}
$$

Analysis code and model outputs are available on the Open Science Foundation platform (Cho, Kizilcec, \& Li, 2021, March 8).

\footnotetext{
1 Tabulation shows the number of participants who checked 'yes' and the percentage distribution across actions.
} 


\section{Results}

4.1 Initial Post-Intervention Beliefs. According to the survey immediately following the intervention, participants thought that $30.06 \%$ of people around the world engage in nature education practices and that $49.87 \%$ of people think that conducting nature education practices is important. Those in the US believed that $35.10 \%$ of people engage in nature education practices, compared to $23.69 \%$ in China $(t(304)=5.33, p<.001)$. US participants also believed that $16.86 \mathrm{pp}$ (percentage points) more people think nature education practices are important $(43.04 \%$ in China vs. $59.90 \%$ in the US, $t(304)=6.25, p<.001)$. Across all participants, the injunctive norm message did not increase approval of nature education $(51.08 \%$ in the control condition vs. $50.95 \%$ in the injunctive condition, $t(368)=$ $-0.04, p=.97)$. The average participant's beliefs about the prevalence of nature education practices were not influenced by the descriptive norm message $(28.12 \%$ vs. $29.16 \%, t(368)=0.39, p=.70)$ or the dynamic norm message $(28.12 \%$ vs. $29.73 \%, t(368)=0.61, p=.54)$. These null results were not subject to moderation by participants' cultural context, especially between China and the US (the prevalence of nature education: $F(3,298)=$ $0.36, p=.78$; the importance of nature education: $F(3,298)=0.79, p=.50)$.

In the same survey, participants also reported that they were highly likely to complete the course $(92.97 \%)$ and conduct nature education practices (87.54\%). The dynamic norm message increased participants' anticipated likelihood of course completion (91.24\% vs. $95.51 \%, t(356)=2.27, p=.024)$, but none of the other norm messages had an effect (descriptive: 90.84\%, $t(356)=-0.17, p=.87$; injunctive: 93.39\%, $t(356)=1.00, p=.32$ ). Compared to Chinese participants, US participants reported a 4.64pp higher likelihood of completing the course $(91.07 \%$ vs. $95.71 \%, t(291)=3.14, p=.002)$ and a $10.33 p p$ higher likelihood of conducting nature education practices $(83.80 \%$ vs. $94.13 \%, t(291)=5.34, p<.001)$. Among Chinese and US participants, culture did not significantly moderate the effects of the social norms interventions on the anticipated likelihood of course completion $(F(3,285)=1.60, p=.19)$ or of conducting nature education $(F(3,285)=0.94, p=.42)$.

4.2 Course Completion. The social norms interventions did not significantly increase the average course completion rate across all participants (descriptive: $b=-.04, t(578)=-.70, p=.49$; dynamic: $b=0.02, t(578)=.37, p=$ .71 ; injunctive: $b=-.03, t(578)=-.48, p=.63)$. However, Figure 2 presents evidence that some intervention effects varied between China and the US $(F(3,464)=2.16, p=.09)$. Among US participants, the injunctive norm intervention increased the completion rate from $51.16 \%$ in the control condition to $71.80 \%(b=.21, t(162)=$ $2.01, p=.046)$. In contrast, neither the descriptive norm intervention $(b=.06, t(162)=.63, p=.53)$ nor the dynamic norm intervention $(b=.11, t(162)=1.05, p=.29)$ significantly increased the completion rate among US participants. The intervention effect estimates for Chinese participants were negative but not statistically significant for all three messages (injunctive: $b=-.12, t(296)=-1.59, p=.11$; descriptive: $b=-.06, t(296)=-.71, p=$ .48 ; dynamic: $b=-.03, t(296)=-.39, p=.70$ ) (See Table 2 for the regression outputs).

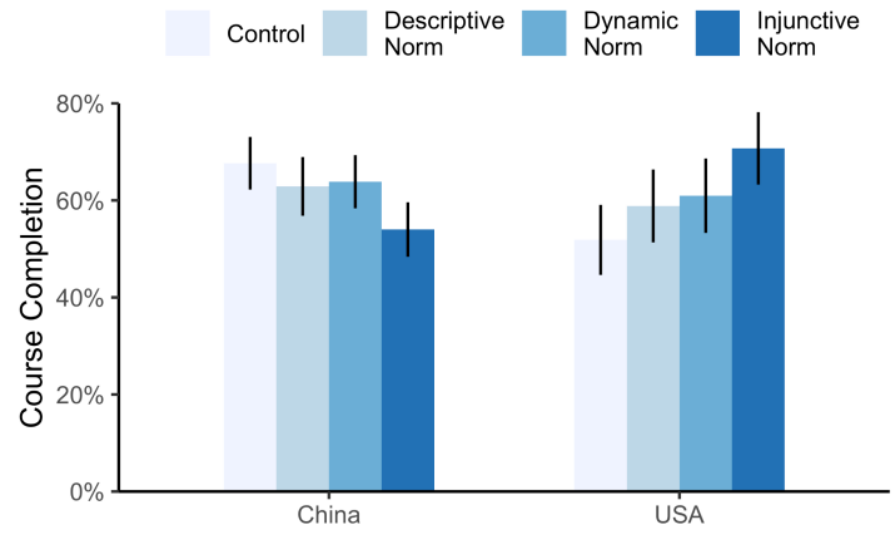

Figure 2. Average Course Completion Rate in Each Experimental Condition Among Chinese and US Course Participants. Point estimates and standard errors are derived from covariate-adjusted regression models.

4.3 Social Course Engagement. Results for social course engagement are similar to those for course completion (Figure 3, Table 2). Overall, the interventions did not significantly increase social course engagement in the course 
(descriptive: $b=-1.45, t(578)=-1.80, p=.07$; dynamic: $b=-.32, t(578)=-.39, p=.70$; injunctive: $b=$ $.11, t(578)=.11, p=.91)$. However, intervention effects varied significantly across participants from China and the US $(F(3,464)=3.20, p=.023)$. Among US participants, the injunctive norm message increased social course engagement by $56 \%$ compared to the control condition $(b=3.52, t(162)=2.24, p=.027)$, while effects of the descriptive norm message $(b=1.47, t(162)=1.36, p=.17)$ and dynamic norm message $(b=2.15, t(162)=$ $1.68, p=.09)$ were smaller and not statistically significant. Chinese participants exhibited $67 \%$ more social course engagement, amounting to 4.34 additional posts on average, compared to US participants in the control condition $(b=$ $4.34, t(464)=3.32, p<.001)$. However, the descriptive norm message reduced Chinese participants' social course engagement by $28 \%$ relative to the control condition $(b=-3.03, t(296)=-2.36, p=.019)$ and effects of the injunctive message $(b=-2.04, t(296)=-1.52, p=.13)$ and dynamic message $(b=-1.79, t(296)=-1.45, p=$ $.15)$ were also negative albeit not statistically significant.

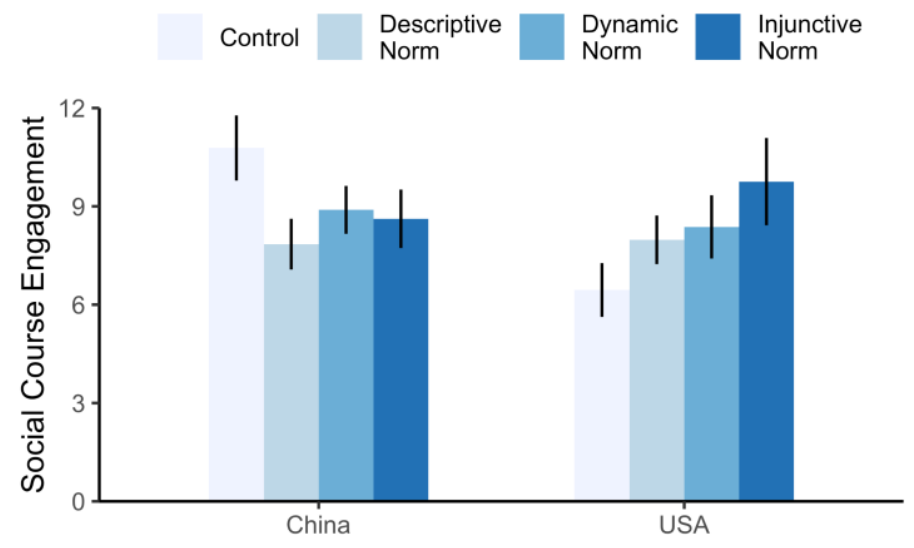

Figure 3. Average Amount of Social Course Engagement in the Course by Experimental Condition Among Chinese and US Participants. Participants' social course engagement is measured by the number of posts, webinar reflections, and comments they contributed in the course. Point estimates and standard errors are derived from covariate-adjusted regression models.

Table 2. Regression outputs for completion rate and social course engagement ${ }^{2}$

\begin{tabular}{lrrrr}
\hline & \multicolumn{2}{c}{ Completion rate } & \multicolumn{2}{c}{ Social course engagement } \\
\hline Sample & U.S. & China & U.S. & China \\
\hline Intercept & $.51^{* * *}$ & $.68^{* * *}$ & $6.26^{* * *}$ & $10.80^{* * *}$ \\
& $(.07)$ & $(.05)$ & $(.79)$ & $(1.01)$ \\
Condition-descriptive & .06 & -.06 & 1.47 & $-3.03^{*}$ \\
& $(.10)$ & $(.08)$ & $(1.08)$ & $(1.28)$ \\
Condition-dynamic & .11 & -.03 & 2.15 & -1.79 \\
& $(.10)$ & $.08)$ & $(1.28)$ & $(1.23)$ \\
Condition-Injunctive & $.21^{*}$ & -.12 & $3.52^{*}$ & -2.04 \\
& $(.10)$ & $(.08)$ & $(1.57)$ & $(1.34)$ \\
Age (cont.) & -.07 & -.01 & -.05 & .54 \\
& $(.03)$ & $(.04)$ & $(.40)$ & $(.52)$ \\
Is female & .06 & .02 & .82 & .22 \\
& $(.04)$ & $(.03)$ & $(.59)$ & $(.45)$ \\
Has Bachelor or higher & .09 & -.01 & .86 & -.17
\end{tabular}

2 The reference group has 0 for all binary variables, the U.S. for country, and the control condition for condition. $* p<.05, * * p<.01, * * * p<.001$. 


\begin{tabular}{lrrrr} 
& $(.05)$ & $(.03)$ & $(.56)$ & $(.36)$ \\
Has work experience in nature & -.04 & -.01 & -.30 & -.43 \\
education & $(.05)$ & $(.03)$ & $(.62)$ & $(.52)$ \\
Knows someone in nature & .04 & .03 & .39 & .70 \\
education & $(.04)$ & $(.03)$ & $(.42)$ & $(.35)$ \\
Is running a program & .02 & -.01 & .56 & -.10 \\
& $(.04)$ & $(.03)$ & $(.55)$ & $(.53)$ \\
\hline Number of observation & 172 & 306 & 172 & 306 \\
Adj. $\mathrm{R}^{2}$ & .04 & -.01 & .02 & .01 \\
\hline
\end{tabular}

4.4 Post-Course Engagement. We collected 131 responses overall (22\% response rate); the majority of responses came from participants who had completed the course (107 out of $356 ; 30 \%$ response rate). Nearly all participants who responded to the follow-up survey implemented or enhanced nature education activities within three months after completing the course. From a set of eleven possible nature education actions (e.g., initiating a new nature education activity), participants took an average of 6.13 actions after completing the Nature Education MOOC $(S D=$ $2.93, \operatorname{Mdn}=7, \operatorname{Min}=0, \operatorname{Max}=11)$ and $97 \%$ reported taking at least one action. Chinese participants did not take significantly more nature education actions than US participants (6.72 vs. $4.84 ; t(83)=-1.57, p=.12)$. The social norms interventions did not significantly increase the number of actions taken after the course (injunctive: $b=$ $-.14, t(119)=-.21, p=.83$; descriptive: $b=.62, t(119)=.86, p=.39$; dynamic: $b=.13, t(119)=.20, p=$ $.84)$. We did not find evidence for variation in treatment effects between China and the US either $(F(3,83)=.74, p=$ .53). US participants did not increase the number of actions taken as a result of the intervention (injunctive: $b=$ $.24, t(33)=.18, p=.86$; descriptive: $b=1.48, t(33)=1.14, p=.26 ;$ dynamic: $b=1.65, t(33)=1.42, p=$ .16). Likewise, there were no significant treatment effects for Chinese participants (injunctive: $b=-.05, t(44)=$ $-.05, p=.96$; descriptive: $b=.96, t(44)=.77, p=.44$; dynamic: $b=-.94, t(44)=-.95, p=.34)$. The followup survey showed that although the interventions did not change nature education outcomes beyond the course, the average level of nature education engagement following the course was notably high.

\section{Discussion}

Social norms interventions have long been utilized as a means of promoting pro-environmental behavior (see Farrow et al., 2017, for a review). The present study examined the effects of descriptive, dynamic, and injunctive norm messages in a Nature Education MOOC designed to support educators in developing nature education activities. We investigated how the interventions impacted course completion rates and social course engagement as proxies for nature education practice (RQ1), how these effects differ between two cultural groups (RQ2), and how much participants have engaged in nature education since taking the course (RQ3). Across all course participants, regardless of their cultural background, we found that the social norm interventions had no effect on course completion or social course engagement or the number of actions taken after the course. We speculate that because participants in the course came from a variety of countries and cultural backgrounds, they have reacted to the social norms interventions in different ways. In fact, we found that in the case of China and the US, their responses canceled each other out to produce an average treatment effect close to zero. Yet the variation in responses in particular to the injunctive norm message, which highlights what people ought to do, provides novel insight into how people in a highly collectivist and tight culture react to a normative message compared to people in a highly individualistic and loose culture. Although the dynamic message was effective in increasing the self-reported anticipated likelihood of completing the course, it did not actually increase course completion or social course engagement in the course. None of the norm messages influenced post-course nature education activities reported in the follow-up survey taken three months after course completion, though the average level of post-course activity was already extremely high, leaving little room for additional activity.

5.1 Cultural Heterogeneity in Intervention Effects. Prior research in cultural psychology suggests that people in different cultures respond differently to normative messages (Fischer, 2006). In collectivist and tight cultures, people tend to be more inclined to conform to normative behaviors compared to those in individualistic and loose cultures (Gelfand, 2012; Gelfand et al., 2017; Trongmateerut \& Sweeney, 2013). It is therefore surprising that none of the 
norm messages had a positive impact on Chinese participants, while some norm messages positively affected US participants. Moreover, our findings for the injunctive norm message effects diverge from prior evidence that injunctive norms are more influential in collectivist than in individualist cultures (Culiberg \& Elgaaied-Gambier, 2016; Walker et al., 2006). In this study, the injunctive norm message did not significantly increase Chinese participants' completion rate or social course engagement, even though it did cause a significant increase in these course outcomes for US participants.

A possible explanation for the difference in the effect of the norm messages between Chinese and US participants is the level of academic pressure in China. In many collectivist countries such as China, learning is perceived to involve competition with other students (Parrish \& Linder-VanBerschot, 2010), and school environments are known to be competitive (H. Li, Meng, Shi, \& Wu, 2012). Students in collectivist countries experience significant pressure from their families to excel academically (Fingerman et al., 2016). In turn, Chinese college students were found to use denial and behavioral disengagement as coping strategies for academic stress more often than US college students (Ying Li, Lindsey, Yin, \& Chen, 2012). Although the end goal of the norm treatment in this study was to promote conducting more nature education activities, the norm message may have signaled competition to Chinese participants and therefore may have activated an academic stressor. As a result, all types of norm messages we tested tended to decrease engagement among Chinese participants. It suggests that the interpretation of the same norm message can vary across cultures and that intervention messages may need to be tailored to the socio-cultural context in which they are deployed to avoid mixed results as we observed in this study, and as other online intervention studies have found (Kizilcec \& Cohen, 2017; Kizilcec et al., 2020).

Another potential explanation for the ineffectiveness of the norm messages for Chinese participants may be that they were unpersuaded that there already exists an established norm, as the field of nature education is a relatively new grassroots movement in China (China Nature Education Network, 2020). As recently as 2014, a group of Chinese environmental organizations started the National Nature Education Forum in response to concerns about children's health and opportunities to access nature (J. Li \& Ernst, 2015; Louv, 2005). Since then, environmental organizations and outdoor clubs began emphasizing nature experiences in their programs, and an increasing number of organizations focusing specifically on nature education have been established as private businesses. This newly emerging field attracts relatively young and inexperienced practitioners with diverse educational backgrounds. In a Chinese national survey of nature education organizations and practitioners, $70 \%$ of the practitioners were younger than 40 years old, and 28\% (61\%) had less than one (three) years of experience in the field (China Nature Education Network, 2020). The population in the current study reflects this trend. On average, compared to US participants, Chinese participants were younger ( 40 vs. 34 years) and less experienced (31\% vs. $13 \%$ with more than five years of nature education experience). Thus, members of this young and dynamic movement may resist normative messages at this stage and perhaps especially injunctive norm messages that tell them what they should do.

5.2 Communicating Norm Messages. Creating effective norm messages for online learning environments like MOOCs poses a number of challenges. First, because course participants come from many different countries, it is complex or even impossible to set up a reference group that is meaningful to all of them. Typically, a reference group is specified in a norm message because group identity is highly associated with the influence of a social norm. People tend to conform to social norms when they identify with the group that originated the social norms (Hornsey, 2008). The norm messages in the present study did not specify to which group of people the norms apply. We made the reference group abstract and decontextualized with expressions such as 'many people' and 'more and more people.' Therefore, the norm messages heavily relied on participants' interpretation of who the message refers to by "people," which may have created heterogeneous effects of the norm messages from participant to participant, and culture to culture.

Second, in a socially opaque environment like a MOOC where participants cannot see how other participants behave in the course, norm messages need to be communicated with a level of abstraction that may have different connotations across cultures. It is difficult to present statistics such as " $70 \%$ of people" for target behaviors like practicing nature education in this context. The norm messages in the present study were therefore written with general quantifiers such as "many" and "millions of people." The term may connote "the majority of people" in the US context, but that may not be the case for other countries. Interpreting such expressions might have been challenging for nonnative English speakers. We used an English norm message with Chinese subtitles, which could also result in different outcomes among participants from the two countries.

Third, planning and implementing nature education practice is a complex behavior that involves multiple components including target audience, learning objectives, educational settings and activities (Yue Li \& Krasny, 2019). Unlike messages such as "reuse towels" (Goldstein et al., 2008) and "order meatless lunch" (Sparkman \& Walton, 2017) used for discrete behaviors, a simple frame "conduct nature education activities" used in our study may 
be too abstract or vague to convey the expected complex actions. We could have included more specific terms in the norm messages such as "taking students to natural areas" and "using five-senses activities to connect students with nature." However, specifying a target action about nature education in the norm message is also difficult, as course participants from around the world employ different teaching techniques based on their local context.

Fourth, the timing of a norm message also determines its effectiveness (Berkowitz, 2004). None of the norm messages we tested affected the number of nature education actions taken by course participants after the course. The primary norm treatment was implemented at the beginning of the course but we also added a reminder in a video towards the end of the course in an attempt to affect post-course outcomes. Given the extremely high levels of postcourse activity, it remains unclear if the interventions had no effect after the course or if there simply was a ceiling effect. We found that the dynamic norm message was most effective for shaping participants' behavioral intentions, independent of their culture. Specifically, immediately after the norm message intervention, the dynamic message was effective in increasing the self-reported anticipated likelihood of completing the course, while the other messages did not. However, the dynamic norms message did not actually increase course completion or social course engagement in the course.

5.3 Evidence of Impact on Subsequent Behavior. Although some studies have examined knowledge transfer from an online course to individuals' professional practice, for instance, in the contexts of data mining (Wang et al., 2014) and functional programming (Chen et al., 2015), it remains relatively unclear how this transfer occurs in communitybased services. Napier and colleagues (2020) surveyed course participants two months after completing a MOOC on school leadership to find out how they applied the course content to improve school leadership to better support student learning. Similarly, the present study conducted a follow-up survey three months after the course ended to measure course participants' implementation of new educational practices in their local communities. We found that among survey respondents (22\% overall response rate and $30 \%$ among course completers), the majority had implemented nature education activities or improved their existing programs. This finding offers further evidence of the real-world effects of large-scale online courses for professional development (Chen et al., 2016; Wang et al., 2014; Napier et al., 2020).

5.4 Limitations. We note limitations that should be considered in the interpretation and application of our findings. First, as in most intervention studies, we are investigating the effects of a specific implementation of a conceptual idea in a specific context. Characteristics of the implementation of our norm messages and of the course context are likely to contribute to the present results. We therefore encourage researchers and practitioners to consider the present set of characteristics when applying or building on our findings. Second, we investigate cultural heterogeneity in treatment effects by comparing the US and China, two large and diverse countries that are frequently used as proxies in crosscultural studies. Nevertheless, we had unequal sample sizes between the two countries in our study, and we acknowledge that there is substantial cultural variation across other collectivist and individualist countries around the world and of course within each of these countries. Third, measuring how online courses influence participant behavior after course completion is relatively new in educational research and still needs more exploration. We used a survey to measure the post-course outcome as other researchers have done in the past (Napier et al., 2020). Most respondents across conditions engaged in nature education activities after taking the course, which may reflect a ceiling effect in our survey measure. To capture differences in behavior, it would be better to ask how much course participants engaged with nature education activities instead of how many different activities they conducted. The survey data may be biased because of socially desirable responses (Steenkamp, De Jong, \& Baumgartner, 2010). Behavioral data, such as publicly available data on community engagement (Chen et al., 2016; Wang et al., 2014), could complement the self-reported survey.

5.5 Future Research. We offer two recommendations for future research in this area. First, further investigations are needed to craft norm messages that can effectively convey a targeted social norm to culturally diverse groups of people. Messages can be tailored by using different languages, adding specific numbers, and setting a relevant reference group such as the course participants themselves, the country, or the specific community that relates to the course content. Second, future research can explore the effect of message framing, especially in Chinese samples. One factor to further investigate is the construal level (abstract vs. concrete framing) of the reference group in the norm message, in light of evidence that people with interdependent self-construals are more likely to attend to concrete information, whereas those with independent self-construals are more likely to attend to abstract information (Spassova \& Lee, 2013). As China is a collectivist culture, where people tend to have interdependent self-construals (Markus \& Kitayama, 1991), normative messages might be more persuasive if they specify a concrete reference group, such as 'Chinese families' instead of 'millions of people.' Finally, to test if the findings also hold in other online 
learning settings, we suggest testing social norms interventions in learning environments other than practice-oriented courses like courses for a cause, which may attract an audience that is more responsive to normative messages. Addressing these issues will help advance our understanding of how to improve real-world outcomes through online courses as an impetus for change.

\section{Conclusion}

The contributions of this study are as follows. First, this study provides a comprehensive field investigation of multiple norm messages in the context of online professional learning to understand which kinds of social norms can promote persistence and engagement during the course, and real-world practice outside of the course. We find that a dynamic norm message was most effective, across different cultures, in raising motivation to complete the course as an immediate intervention effect. However, the effect of the dynamic norm message did not last long: it did not increase course participants' engagement during the course, completion rate, or actions outside of the course. Second, this study is a rare cross-cultural look at how social norm messages differ in their effects across two cultural contexts. It demonstrates that an injunctive norm message is effective in promoting course persistence for participants in the individualist US, but not for participants in collectivist China. Third, the present study offers empirical evidence for how online courses can contribute to real-world professional development, finding that participants are highly motivated and engaged weeks after taking the course. This finding highlights the potential of courses for a cause as a vehicle to facilitate community-based practice at scale.

\section{References}

Anderson, A., Huttenlocher, D., Kleinberg, J., \& Leskovec, J. (2014). Engaging with massive online courses. In Proceedings of the 23rd International Conference on World Wide Web (pp. 687-698), Seoul, Korea. https://doi.org/10.1145/2566486.2568042.

Baiocchi, M., \& Kizilcec, R. F. (2018). Criteria-based randomization: Explicit and exact control in multi-arm trials. Available at SSRN 3339748.

Anderson, A., Huttenlocher, D., Kleinberg, J., \& Leskovec, J. (2014). Engaging with massive online courses. In Proceedings of the 23rd International Conference on World Wide Web (pp. 687-698), Seoul, Korea. https://doi.org/10.1145/2566486.2568042.

Baiocchi, M., \& Kizilcec, R. F. (2018). Criteria-based randomization: Explicit and exact control in multi-arm trials. Available at SSRN 3339748.

Barak, M., \& Watted, A. (2017). Project-Based MOOC: Enhancing knowledge construction and motivation to learn. In Digital tools and solutions for inquiry-based STEM learning (pp. 282-307): IGI Global.

Berkowitz, A. D. (2004). The social norms approach: Theory, research, and annotated bibliography. Retrieved from http://www.alanberkowitz.com/articles/social_norms.pdf.

Bresnahan, M. J., \& Zhuang, J. (2016). Effects of normative antismoking messages in China-a brief report. Health Communication, 31(3), 374-378. doi:10.1080/10410236.2014.952804.

Brinton, C. G., Chiang, M., Jain, S., Lam, H., Liu, Z., \& Wong, F. M. F. (2014). Learning about social learning in MOOCs: From statistical analysis to generative model. IEEE Transactions on Learning Technologies, 7(4), 346-359. doi:10.1109/TLT.2014.2337900.

Chen, G., Davis, D., Hauff, C., \& Houben, G.-J. (2016). Learning transfer: Does it take place in MOOCs? An investigation into the uptake of functional programming in practice. In Proceedings of the 3rd ACM Conference on Learning@ Scale (pp. 409-418).

Cho, J., Kizilcec, R. F., \& Li, Y. (2021, March 8). Online Learning and Social Norms: Evidence from a CrossCultural Field Experiment in a Course for a Cause. https://doi.org/10.17605/OSF.IO/CYZNE

Children \& Nature Network. (2020). Nature-based Teaching Certificate Course. Retrieved from https://naaee.org/eepro/learning/online-courses/nature-based-teaching-certificate-course.

China Nature Education Network. (2020). National Development of Nature Education in China. Retrieved from http://www.natureeducation.org.cn/.

Cialdini, R. B. (2003). Crafting normative messages to protect the environment. Current Directions in Psychological Science, 12(4), 105-109. doi:10.1111/1467-8721.01242. 
Cialdini, R. B. (2009). Influence: Science and practice (Vol. 4). Boston, MA: Pearson Education

Cialdini, R. B., Demaine, L. J., Sagarin, B. J., Barrett, D. W., Rhoads, K., \& Winter, P. L. (2006). Managing social norms for persuasive impact. Social Influence, 1(1), 3-15. doi:10.1080/15534510500181459.

Clinnin, K. (2014). Redefining the MOOC: Examining the multilingual and community potential of massive online courses. Journal of Global Literacies, Technologies, and Emerging Pedagogies, 2(3), 140-162.

Coetzee, D., Fox, A., Hearst, M. A., \& Hartmann, B. (2014, February). Should your MOOC forum use a reputation system? In Proceedings of the 17th ACM conference on Computer supported cooperative work \& social computing (pp. 1176-1187).

Crawford, J., Butler-Henderson, K., Rudolph, J., Malkawi, B., Glowatz, M., Burton, R., . . Lam, S. (2020). COVID-19: 20 countries' higher education intra-period digital pedagogy responses. Journal of Applied Learning \& Teaching, 3(1), 1-20. doi:10.37074/jalt.2020.3.1.7.

Culiberg, B., \& Elgaaied-Gambier, L. (2016). Going green to fit in - understanding the impact of social norms on pro-environmental behaviour, a cross-cultural approach. International Journal of Consumer Studies, 40(2), 179-185. doi:10.1111/ijcs.12241.

Davis, D., Jivet, I., Kizilcec, R. F., Chen, G., Hauff, C., \& Houben, G. J. (2017, March). Follow the successful crowd: raising MOOC completion rates through social comparison at scale. In Proceedings of the seventh international learning analytics \& knowledge conference (pp. 454-463).

DeBoer, J., Ho, A. D., Stump, G. S., \& Breslow, L. (2014). Changing "course": Reconceptualizing educational variables for massive open online courses. Educational Researcher, 43(2), 74-84. doi:10.3102/0013189x14523038.

DuBois, B., Krasny, M. E., \& Russ, A. (2019). Online professional development for environmental educators: strategies to foster critical thinking and social interactions. Environmental Education Research, 1-15. doi:10.1080/13504622.2018.1564247.

Eisenberg, M., \& Fischer, G. (2014). MOOCs: A perspective from the learning sciences. In L. J. Polman, E. A. Kyza, D. K. O’Neill, I. Tabak, W. R. Penuel, A. S. Jurow, K. O'Connor, T. Lee, \& L. D’Amico (Eds.), Learning and Becoming in Practice: 11th International Conference of the Learning Sciences (ICLS) 2014. Boulder, CO.: International Society of the Learning Sciences.

Farrow, K., Grolleau, G., \& Ibanez, L. (2017). Social norms and pro-environmental behavior: A review of the evidence. Ecological economics, 140, 1-13. doi:10.1016/j.ecolecon.2017.04.017.

Fingerman, K. L., Cheng, Y.-P., Kim, K., Fung, H. H., Han, G., Lang, F. R., . . Wagner, J. (2016). Parental Involvement With College Students in Germany, Hong Kong, Korea, and the United States. Journal of Family Issues, 37(10), 1384-1411. doi:10.1177/0192513x14541444.

Fischer, R. (2006). Congruence and functions of personal and cultural values: Do my values reflect my culture's values? Personality and Social Psychology Bulletin, 32(11), 1419-1431. doi:10.1177/0146167206291425.

Formanek, M., Wenger, M. C., Buxner, S. R., Impey, C. D., \& Sonam, T. (2017). Insights about large-scale online peer assessment from an analysis of an astronomy MOOC. Computers \& Education, 113, 243-262. doi:10.1016/j.compedu.2017.05.019.

Gelfand, M. J. (2012). Culture's constraints: International differences in the strength of social norms. Current Directions in Psychological Science, 21(6), 420-424. doi:10.1177/0963721412460048.

Gelfand, M. J., Harrington, J. R., \& Jackson, J. C. (2017). The strength of social norms across human groups. Perspectives on Psychological Science, 12(5), 800-809. doi:10.1177/1745691617708631.

Göckeritz, S., Schultz, P. W., Rendón, T., Cialdini, R. B., Goldstein, N. J., \& Griskevicius, V. (2010). Descriptive normative beliefs and conservation behavior: The moderating roles of personal involvement and injunctive normative beliefs. European Journal of Social Psychology, 40(3), 514-523. doi:10.1002/ejsp.643.

Goldstein, N. J., Cialdini, R. B., \& Griskevicius, V. (2008). A room with a viewpoint: Using social norms to motivate environmental conservation in hotels. Journal of Consumer Research, 35(3), 472-482. doi:10.1086/586910.

Green, M., \& Somerville, M. (2015). Sustainability education: Researching practice in primary schools. Environmental Education Research, 21(6), 832-845. doi:10.1080/13504622.2014.923382. 
Győrffy, Z., Birkás, E., \& Sándor, I. (2016). Career motivation and burnout among medical students in Hungary could altruism be a protection factor? BMC Medical Education, 16(1), 182. doi:10.1186/s12909-016-0690-5.

Henrich, J., Heine, S. J., \& Norenzayan, A. (2010). Most people are not WEIRD. Nature, 466(7302), 29-29. doi:10.1038/466029a.

Higgs, S., Liu, J., Collins, E. I. M., \& Thomas, J. M. (2019). Using social norms to encourage healthier eating. Nutrition Bulletin, 44(1), 43-52. doi:10.1111/nbu.12371.

Hofstede, G. (1980). Culture and Organizations. International Studies of Management \& Organization, 10(4), 1541. doi:10.1080/00208825.1980.11656300.

Hornsey, M. J. (2008). Social identity theory and self-categorization theory: A historical review. Social and Personality Psychology Compass, 2(1), 204-222. doi:10.1111/j.1751-9004.2007.00066.x.

Joyner, D. A., Wang, Q., Thakare, S., Jing, S., Goel, A., \& MacIntyre, B. (2020). The synchronicity paradox in online education. In Proceedings of 7th ACM Conference on Learning@ Scale (pp. 15-24). doi: 10.1145/3386527.3405922.

Kahan, T., Soffer, T., \& Nachmias, R. (2017). Types of participant behavior in a massive open online course. International Review of Research in Open and Distributed Learning: IRRODL, 18(6), 1-18. doi:10.19173/irrodl.v18i6.3087.

Kellogg, S. B., Booth, S., \& Oliver, K. (2014). A social network perspective on peer supported learning in MOOCs for educators. The International Review of Research in Open and Distributed Learning, 15(5), 263-289. doi: 10.19173/irrodl.v15i5.1852.

Kizilcec, R. F., \& Cohen, G. L. (2017). Eight-minute self-regulation intervention raises educational attainment at scale in individualist but not collectivist cultures. In Proceedings of the National Academy of Sciences, 114, 4348-4353. doi:10.1073/pnas.1611898114

Kizilcec, R. F., \& Halawa, S. (2015). Attrition and achievement gaps in online learning. In Proceedings of the 2nd ACM Conference on Learning @ Scale (pp. 57-66), Vancouver, BC, Canada. doi:10.1145/2724660.2724680.

Kizilcec, R. F., Makridis, C. A., \& Sadowski, K. C. (2021). Pandemic response policies' democratizing effects on

online learning. Proceedings of the National Academy of Sciences, 118(11). doi: 10.1073/pnas.2026725118

Kizilcec, R. F., Reich, J., Yeomans, M., Dann, C., Brunskill, E., Lopez, G., . . Tingley, D. (2020). Scaling up behavioral science interventions in online education. In Proceedings of the National Academy of Sciences, 117, 14900-14905. doi: 10.1073/pnas.1921417117.

Kizilcec, R. F., Saltarelli, A. J., Reich, J., \& Cohen, G. L. (2017). Closing global achievement gaps in MOOCs. Science, 355(6322), 251-252. doi: 10.1126/science.aag2063.

Kizilcec, R. F., \& Schneider, E. (2015). Motivation as a lens to understand online learners: Toward data-driven design with the OLEI scale. ACM Transactions on Computer-Human Interaction (TOCHI), 22(2), 1-24.

Kizilcec, R. F., Schneider, E., Cohen, G. L., \& McFarland, D. A. (2014). Encouraging forum participation in online courses with collectivist, individualist and neutral motivational framings. eLearning Papers, 37, 13-22.

Krasny, M. E., DuBois, B., Adameit, M., Atiogbe, R., Baih, L., Bold-erdene, T., . . Yao, Y. (2018). Small Groups in a Social Learning MOOC (slMOOC): Strategies for Fostering Learning and Knowledge Creation. 2018, 22(2). doi:10.24059/olj.v22i2.1339.

Krasny, M. E., Golshani, Z., Hampton, B. L., Coleman, J. F. R. M., Schrenk, M., Warakula, M. R., . . Yu, Y. (2019). MOOC contributions to a "better place for all". In K. Zhang, C. J. Bonk, T. C. Reeves, \& T. H. Reynolds (Eds.), MOOCs and Open Education in the Global South: Challenges, Successes, and Opportunities. New York: Routledge.

Li, H., Meng, L., Shi, X., \& Wu, B. (2012). Does attending elite colleges pay in China? Journal of Comparative Economics, 40(1), 78-88. doi:10.1016/j.jce.2011.10.001.

Li, J., \& Ernst, J. (2015). Exploring value orientations toward the human-nature relationship: a comparison of urban youth in Minnesota, USA and Guangdong, China. Environmental Education Research, 21(4), 556-585. doi:10.1080/13504622.2014.910499.

Li, Y., \& Krasny, M. E. (2019). Practice change in environmental education: Lessons from professional development. Environmental Education Research, 25(7): 1119-1136. doi:10.1080/13504622.2018.1540033. 
Li, Y., Lindsey, B. J., Yin, X., \& Chen, W. (2012). A comparison of American and Chinese students' perceived stress, coping styles, and health promotion practices. Journal of Student Affairs Research and Practice, 49(2), 211-227. doi:10.1515/jsarp-2012-6298.

Loschelder, D. D., Siepelmeyer, H., Fischer, D., \& Rubel, J. A. (2019). Dynamic norms drive sustainable consumption: Norm-based nudging helps café customers to avoid disposable to-go-cups. Journal of Economic Psychology, 75, 102146. doi:10.1016/j.joep.2019.02.002.

Louv, R. (2005). Last child in the woods: Saving our children from nature-deficit disorder. Chapel Hill, NC: Algonquin books.

Markus, H. R., \& Kitayama, S. (1991). Culture and the self: Implications for cognition, emotion, and motivation. Psychological review, 98(2), 224.

Mortensen, C. R., Neel, R., Cialdini, R. B., Jaeger, C. M., Jacobson, R. P., \& Ringel, M. M. (2019). Trending Norms: A Lever for Encouraging Behaviors Performed by the Minority. Social Psychological and Personality Science, 10(2), 201-210. doi:10.1177/1948550617734615.

$\mathrm{Mu}$, Y., Kitayama, S., Han, S., \& Gelfand, M. J. (2015). How culture gets embrained: Cultural differences in eventrelated potentials of social norm violations. In Proceedings of the National Academy of Sciences, 112(50), 15348-15353. doi:10.1073/pnas.1509839112.

Napier, A., Huttner-Loan, E., \& Reich, J. (2020). Evaluating learning transfer from MOOCs to workplaces: A case study from teacher education and launching innovation in schools. RIED. Revista Iberoamericana de Educación a Distancia, 23(2), 45-64. doi:10.5944/ried.23.2.26377.

Nomura, H., John, P. C., \& Cotterill, S. (2011). The use of feedback to enhance environmental outcomes: a randomised controlled trial of a food waste scheme. Local Environment, 16(7), 637-653. doi:10.1080/13549839.2011.586026.

Oh, S. H. (2013). Do collectivists conform more than individualists? Cross-cultural differences in compliance and internalization. Social Behavior and Personality: an international journal, 41(6), 981-994. doi:10.2224/sbp.2013.41.6.981.

Parrish, P., \& Linder-VanBerschot, J. (2010). Cultural dimensions of learning: Addressing the challenges of multicultural instruction. The International Review of Research in Open and Distributed Learning, 11(2), 1-19. doi:10.19173/irrodl.v11i2.809.

Skrypnyk, O., Joksimović, S. k., Kovanović, V., Gašević, D., \& Dawson, S. (2015). Roles of course facilitators, learners, and technology in the flow of information of a cMOOC. The International Review of Research in Open and Distributed Learning, 16(3). doi:10.19173/irrodl.v16i3.2170.

Smith, J. R., Louis, W. R., Terry, D. J., Greenaway, K. H., Clarke, M. R., \& Cheng, X. (2012). Congruent or conflicted? The impact of injunctive and descriptive norms on environmental intentions. Journal of Environmental Psychology, 32(4), 353-361. doi:10.1016/j.jenvp.2012.06.001.

Sparkman, G., \& Walton, G. M. (2017). Dynamic norms promote sustainable behavior, even if it is counternormative. Psychological Science, 28(11), 1663-1674. doi:10.1177/0956797617719950.

Spassova, G., \& Lee, A. Y. (2013). Looking into the future: A match between self-view and temporal distance. Journal of Consumer Research, 40(1), 159-171. https://doi.org/10.1086/669145

Spoelstra, H., Van Rosmalen, P., \& Sloep, P. (2014). Toward project-based learning and team formation in open learning environments. Journal of Universal Computer Science, 20(1), 57-76.

Steenkamp, J. B. E., De Jong, M. G., \& Baumgartner, H. (2010). Socially desirable response tendencies in survey research. Journal of Marketing Research, 47(2), 199-214.

Triandis, H. C. (1995). Individualism and collectivism. Boulder, CO: Westview Press.

Trongmateerut, P., \& Sweeney, J. T. (2013). The influence of subjective norms on whistle-blowing: A cross-cultural investigation. Journal of Business Ethics, 112(3), 437-451. doi:10.1007/s10551-012-1270-1.

Tyers, R. (2018). Nudging the jetset to offset: voluntary carbon offsetting and the limits to nudging. Journal of Sustainable Tourism, 26(10), 1668-1686. doi:10.1080/09669582.2018.1494737.

Walker, G. J., Courneya, K. S., \& Deng, J. (2006). Ethnicity, gender, and the theory of planned behavior: The case of playing the lottery. Journal of Leisure Research, 38(2), 224-248. doi:10.1080/00222216.2006.11950077. 
Wang, Y., Paquette, L., \& Baker, R. (2014). A longitudinal study on learner career advancement in MOOCs. Journal of Learning Analytics, 1(3), 203-206.

Zhang, J., Skryabin, M., \& Song, X. (2016). Understanding the dynamics of MOOC discussion forums with simulation investigation for empirical network analysis (SIENA). Distance Education, 37(3), 270-286. doi:10.1080/01587919.2016.1226230. 


\section{Appendix A. Examples of Final Projects in English and Chinese}
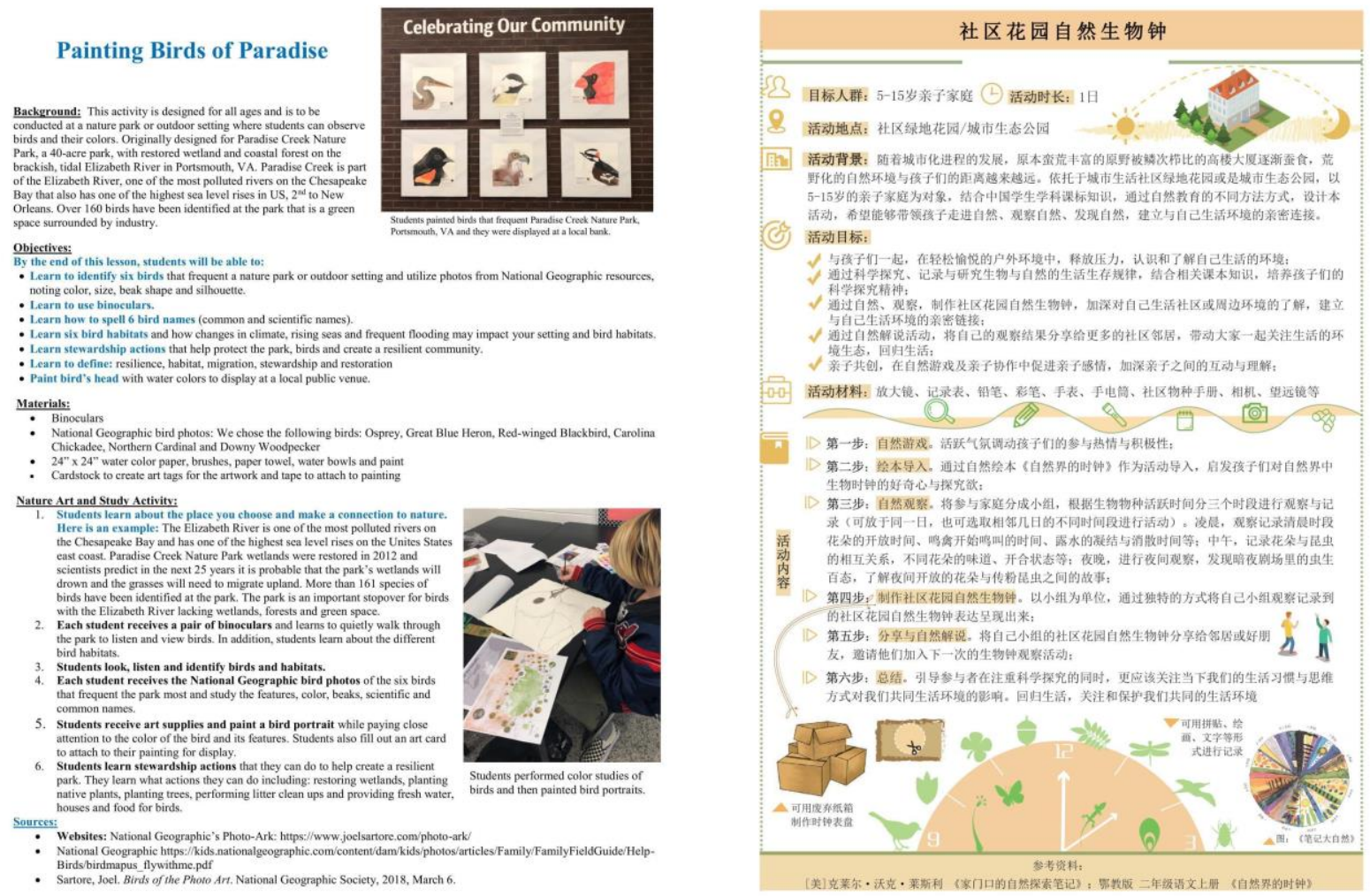